\title{
Author Correction: Immune reconstitution therapies: concepts for durable remission in multiple sclerosis
}

Jan D. Lünemann (10), Tobias Ruck, Paolo A. Muraro (1), Amit Bar-Or and Heinz Wiendl

Nature Reviews Neurology (2019) https://doi.org/10.1038/s41582-019-0268-z Published online 24 October 2019

In the originally published article, in the author list, Amit Bar-Or's name was spelt incorrectly. The error has been corrected for the HTML and PDF versions of the article.

https://doi.org/10.1038/s41582-020-0310-1 I Published online 14 January 2020

(c) Springer Nature Limited 2020 